\title{
Cooling the dark energy camera instrument
}

\author{
R.L. Schmitt ${ }^{\text {a }}$, H. Cease ${ }^{\text {a }}$, D. DePoy ${ }^{\text {c }}$, H. T. Diehl ${ }^{\mathrm{a}}$, J. Estrada ${ }^{\mathrm{a}}$, B. Flaugher ${ }^{\mathrm{a}}$, \\ S. Kuhlmann ${ }^{\mathrm{b}}$, Birce Onal ${ }^{\mathrm{a}}$, A. Stefanik ${ }^{\mathrm{a}}$ \\ ${ }^{a}$ Fermi National Accelerator Laboratory ${ }^{2}$, Box 500, Batavia, IL, USA 60510 \\ ${ }^{\mathrm{b}}$ Argonne National Laboratory, Argonne, IL, USA 60439 \\ 'Ohio State University
}

For the Dark Energy Survey Collaboration

\begin{abstract}
DECam, camera for the Dark Energy Survey (DES), is undergoing general design and component testing. For an overview see DePoy, et al in these proceedings. For a description of the imager, see Cease, et al in these proceedings. The CCD instrument will be mounted at the prime focus of the CTIO Blanco 4m telescope. The instrument temperature will be $173 \mathrm{~K}$ with a heat load of $113 \mathrm{~W}$. In similar applications, cooling CCD instruments at the prime focus has been accomplished by three general methods. Liquid nitrogen reservoirs have been constructed to operate in any orientation, pulse tube cryocoolers have been used when tilt angles are limited and Joule-Thompson or Stirling cryocoolers have been used with smaller heat loads. Gifford-MacMahon cooling has been used at the Cassegrain but not at the prime focus. For DES, the combined requirements of high heat load, temperature stability, low vibration, operation in any orientation, liquid nitrogen cost and limited space available led to the design of a pumped, closed loop, circulating nitrogen system. At zenith the instrument will be twelve meters above the pump/cryocooler station. This cooling system expected to have a 10,000 hour maintenance interval. This paper will describe the engineering basis including the thermal model, unbalanced forces, cooldown time, the single and two-phase flow model.
\end{abstract}

Keyword list: DES, DECAM, CTIO, NOAO, CCD, liquid nitrogen, pump, camera cooling

\section{Introduction}

DECam will use an extremely red sensitive 520 Megapixel camera, a 1 meter diameter, 2.2 degree field of view prime focus corrector, and a data acquisition system fast enough to take images in 17 seconds. The cage containing the system mounts at the prime focus of the Blanco 4-meter telescope at CERRO TOLOLO INTER-AMERICAN OBSERVATORY (CTIO), a southern hemisphere National Optical Astronomy Observatory (NOAO) telescope.. It will be used to conduct the a large scale sky survey called the Dark Energy Survey.

Over five years it will use $30 \%$ of the available time on the telescope to pursue a high precision multi-bandpass wide area survey, designed to produce photometric redshifts from $0.2<\mathrm{z}<1.3$. The survey g,r,i,z data will cover 5000 sqdegrees, with 4000 sq-degrees overlapping the Sunyaev-Zeldovich CMB survey being conducted by the South Pole Telescope. The four science goals aim at extracting cosmological information on the dark energy from 1) cluster

${ }^{*}$ RLSchmitt@fnal.gov, 1-630-840-4849. Work supported by the U.S. Department of Energy under contract No. DE-AC02$07 \mathrm{CH} 11359$. 
counting and spatial distribution of clusters at $0.1<\mathrm{z}<1.3,2$ ) the shifting of the galaxy spatial angular power spectra with redshift, 3) weak lensing measurements on several redshift shells to $z \sim 1$, and 4) 2000 supernovae at $0.3<z<0.8$. 


\section{Cooling System Design Requirements}

\subsection{Camera design requirements}

- Expected range of mean focal plane temperature: $-120^{\circ} \mathrm{C}$ to $-80^{\circ} \mathrm{C}$

- The detector focal plane spatial temperature variation will be $\leq 10$ degrees $\mathrm{K}$ across the focal plane

- Detector temperature stability will be \pm 0.25 degree K stability over 12 hours

- External surfaces of DECam will be $<3^{\circ} \mathrm{C}$ warmer than ambient temperature

- All instrument components shall perform to specification at all possible orientations

- DECam and associated structures will block $<3 \%$ of the light that would otherwise reach the primary mirror

- Dewar vacuum must be $<2 \times 10^{-4}$ torr before cooling below ambient

- Dewar vacuum should be $<10^{-5}$ torr in normal operation

- Temperature/vacuum requirements should be met with no manual input more frequently than once per $\sim 30$ hours

- Vacuum and focal plane temperature should be maintained continuously over a period of $>12$ months without interruption

- The focal plane should come to operational temperature in $<8$ hours

- The dewar should warm up and be ready to open to ambient environment in $<12$ hours

- The dewar and associated cooling and vacuum system will not distort or otherwise move the focal plane by more than $15 \mu \mathrm{m}$ in $\mathrm{x}$ or $\mathrm{y}$

- The dewar and associated cooling and vacuum system will not distort the flatness of the focal plane by more than $15 \mu \mathrm{m}$

- The dewar and associated cooling and vacuum system will not vibrate the focal plane by more than $1.5 \mu \mathrm{m}$ at any frequency

- Outdoor temperature -5 to $27 \mathrm{C}$

- Altitude $2200 \mathrm{~m}$ above sea level, $77 \mathrm{kPa}$ 


\subsection{Imager Heat Load}

Heat loads for the Imager are summarized in table 1. Calculated heat loads for the focal plate have been confirmed by testing. Heat loads for the nitrogen circulation system are summarized in Table 2 . They are based on calculations and will be tested in 2008 . The cryocooler chosen to refrigerate this system has a capacity of 360 watts at an operating temperature of $90 \mathrm{~K}$. The cryo-cooler has $40 \%$ more capacity than what is required in the steady state operating condition.

Table 1. Summary of heat Loads

\begin{tabular}{|l|l|l|}
\hline \multirow{2}{*}{ Item } & $\begin{array}{l}\text { Warm Night } \\
\text { CCD Temp. }-100{ }^{\circ} \mathrm{C} \\
\text { Ambient } 27{ }^{\circ} \mathrm{C}\end{array}$ \\
\hline Focal Plate & Thermal Radiation & 43.0 Watts \\
\cline { 2 - 3 } & Conductivity Supports & 0.67 Watts \\
\hline \multirow{3}{*}{$\begin{array}{l}\text { ECD } \\
\text { Electronics }\end{array}$} & Conductivity cables & 10.3 Watts \\
\cline { 2 - 3 } & CCD JFET (70 CCDs) & 0.6 Watts \\
\cline { 2 - 3 } & $\begin{array}{l}\text { CCD output amplifier } \\
\text { (70 CCDs) }\end{array}$ & 2.8 Watts \\
\cline { 2 - 3 } & VIB Interface Card Amps. & 28 Watts \\
\hline $\begin{array}{l}\text { Thermal } \\
\text { Control }\end{array}$ & Trim Heaters & 19 Watts \\
\hline $\begin{array}{l}\text { Heat } \\
\text { Exchanger }\end{array}$ & Radiation & 7.6 Watts \\
\cline { 2 - 3 } Imager Total & 1.0 Watts \\
\hline
\end{tabular}

Table 2, Cooling System heat loads

\begin{tabular}{|c|c|}
\hline Item & \\
\hline Transfer lines & 65 Watts \\
\hline Valves and fittings & 16 Watts \\
\hline Circulation pump & 38 Watts \\
\hline 200 liter reservoir & 25 Watts \\
\hline Imager total & 113 Watts \\
\hline System total & $\mathbf{2 5 7}$ Watts \\
\hline
\end{tabular}

\section{Cooling strategy}

\subsection{Cooling strategies for instruments similar to DECam}

Table 3 shows a selection of existing, nearing completion, and planned instruments and surveys that are similar to $\mathrm{DES} / \mathrm{DECam}$. The list is not complete; there are many $8 \mathrm{~K} \times 8 \mathrm{~K}$ CCD cameras on telescope, for example. However, the list includes many state-of-the-art cameras existing on or planned for major ground-based telescopes and new facilities. Table 3 also gives the cooling strategies adopted by the various instruments along with a rough estimate of the cooling power needed relative to DECam (based on the physical size of the focal plane). The information in Table 3 was gathered off of web pages and recent presentations for the various projects. A previous or planned instance using Gifford-McMahon cryocoolers at the prime focus has not been found. 
Table 3: Wide-Field CCD Cameras Cooling Strategy

\begin{tabular}{|c|c|c|c|c|c|}
\hline INSTRUMENT & $\begin{array}{c}\text { \# OF } \\
\text { PIXELS }\end{array}$ & $\begin{array}{l}\text { FOCAL } \\
\text { PLANE } \\
\text { AREA }\end{array}$ & $\begin{array}{l}\text { HEAT } \\
\text { LOAD }\end{array}$ & COOLING APPROACH & $\begin{array}{c}\text { MOUNT } \\
\text { LOCATION }\end{array}$ \\
\hline & (M) & (relative) & $(W)$ & & \\
\hline MOSAIC & & & & IN fond fillod & \\
\hline MUSAIC & 64 & 0.12 & & $\mathrm{LN}_{2}$ (hand filled) & prime \\
\hline CFHT Megacam & 380 & 0.59 & & Pulse tube + Thermal capacitor & prime \\
\hline MMT Megacam & 340 & 0.52 & & $\mathrm{LN}_{2}$ (hand filled) & cassegrain \\
\hline SuprimeCam & 84 & 0.16 & 11 & Stirling cycle & prime \\
\hline WIYN ODI & 1024 & 1.57 & 146 & $\begin{array}{l}\text { Gifford-McMahon ( } 2 \text { heads) } \\
\text { with vibration isolation }\end{array}$ & Nasymth \\
\hline VST OmegaCam & 256 & 0.49 & & $\mathrm{LN}_{2}$ (not clear how filled) & cassegrain \\
\hline Pan-Starrs & 1475 & 1.89 & & Still looking & cassegrain \\
\hline DECam & 520 & 1 & 113 & Recirculating, high-pressure $\mathrm{LN}_{2}$ & prime \\
\hline
\end{tabular}

\subsection{Cooling System Selection for DECam}

A variety of cooling systems were considered as shown in Figure 1. The approach was to consider any reasonable cooling system that could meet the requirements. Besides cooling power other considerations included temperature stability, operating cost, vibration and size. While specific dimensions are not specified in the thermal specifications, the overall Imager Vessel has limited space. The cooling system must fit in that space along with all the other components. The advantages and disadvantages of each cooling scheme were considered leading to the decision to circulate liquid nitrogen.

Figure 1 Cooling system decision tree

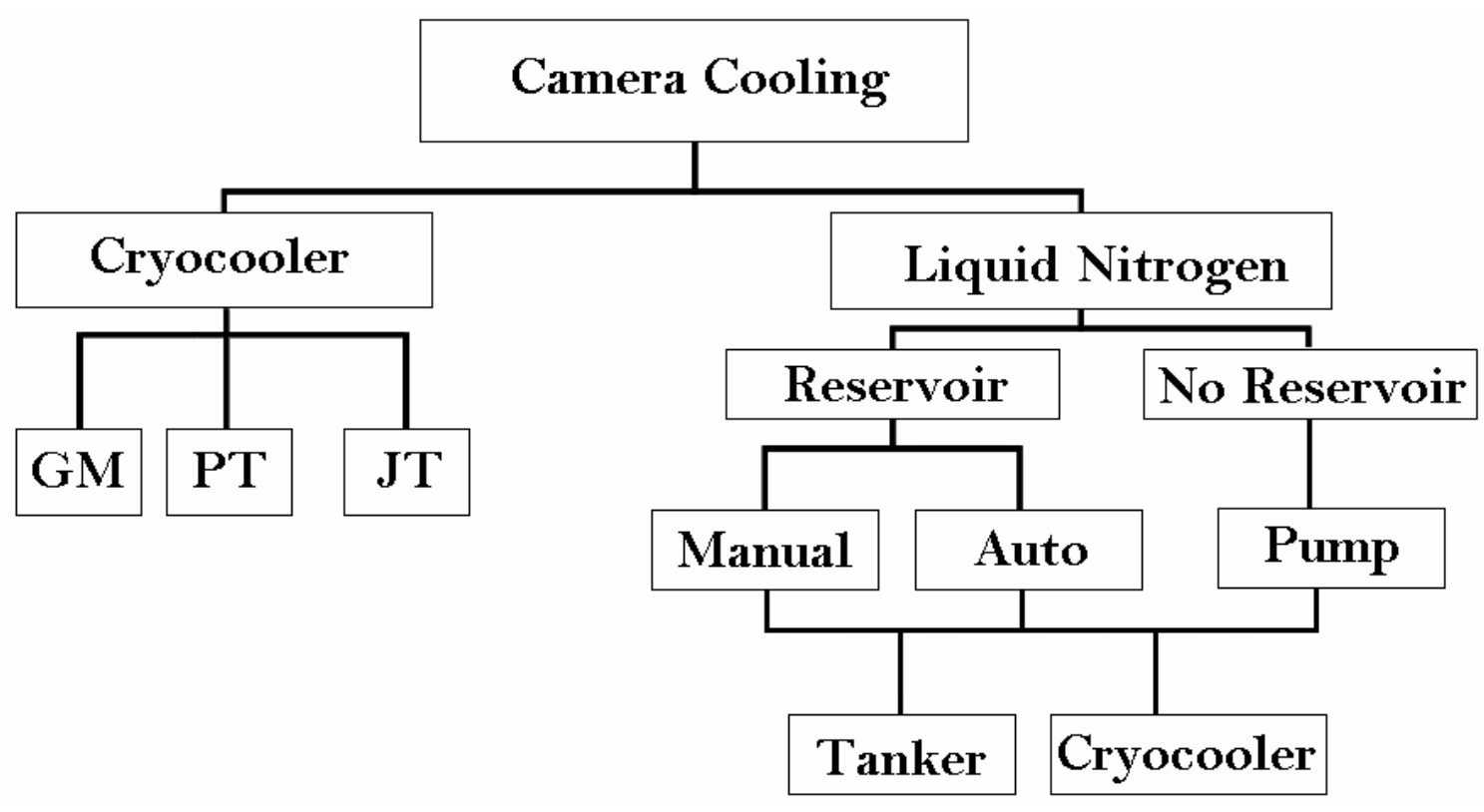




\subsection{Gifford McMahon cryocooler mounted on the camera}

Mounting cryocoolers on the camera offers advantages of minimizing parasitic heat load, un-insulated hoses, simple temperature control and simple operation. However each type of cryocooler has disadvantages that make them unsuitable for DECam.

G-M (Gifford-McMahon) cryocoolers are available with adequate cooling capacity and can operate in any orientation. But vibration is substantial and difficult to quantify. There are lower frequency unbalanced forces due to piston motion and higher frequency forces due to gas flow through the valve. Normal G-M practice at Fermilab and elsewhere is to carry unbalanced forces back to a substantial structure away from the sensitive detectors and use a flexible connection to conduct heat to the cryocooler. For DECam the forces would be carried back to the telescope frame. CSA Engineering reported that they have developed an active means of damping cryocooler vibrations. These were Stirling coolers on aircraft mounted systems. Vibrations have been reduced by a factor of one hundred with Stirling coolers, which operate at $50 \mathrm{~Hz}$ and have a repeatable vibration pattern. Damping G-M cryocoolers is more difficult because: a) the gas inrush is turbulent flow, very complex and variable, b) it is difficult to make an support that is both stiff enough to support the weight without sagging and soft enough to adsorb low frequency vibrations. ${ }^{1}$ Prime focus telescopes have different vibration issues than Cassegrain telescopes. At ground based experiments such as Laser Interferometer Gravitational Wave Observatory (LIGO), Cryogenic Dark Matter Search (CDMS), and at High Energy Accelerator Research Organization (KEK) vibration isolation is a major concern. In those cases the unbalanced forces are carried back to earth and the sensitive instruments are isolated by flexible conductors. Those cryocoolers have a fixed orientation so the weight can be carefully balanced. Vibration is very case-dependant. A substantial engineering effort could be spent studying vibration and still there would be no confidence that it would work until the instrument was mounted on the telescope. We chose to put the cryocooler on the ground because this is less risky and requires less effort than mounting it on the instrument.

\subsection{Pulse Tube cryocooler}

Pulse tube cryocoolers are available with adequate cooling capacity when operated vertically, but their capacity seriously degrades when tilted. CFHT Megacam overcame this limitation by using a large themal mass to carry the heat load during intermittent tilted orientations. The DECam requirement to operate at any orientation ruled out the use of pulse tube cryocoolers.

\subsection{Joule-Thompson cryocooler}

Joule-Thompson (JT) cryocoolers have low vibration and are orientation independent. However there were no commercial units available with adequate cooling power. Multiple units would be required, crowding the limited space available. In addition their cooling power decreased both above and below a peak performance temperature and would therefore require a long cooldown time. Their relatively warm operating temperature would require heavy thermal links between the cryocoolers and the focal plate, adding weight and stiffness to the supports. These cryocoolers have some advantages if the right model was available. Searches were made for commercial units and experts both inside and outside the collaboration were consulted.

- Del Allspach, cryocooler expert at Fermilab, found no suitable commercial units

- Christoph Haberstroh, cryocooler expert under contract with Fermilab, found no commercial units and recommends using liquid nitrogen dewars

- Dwight Richeimer of Praxair offers no commercial units and recommends circulating liquid or gaseous nitrogen

- Polycold has large units but not cold enough

- Fermilab could design a JT cooling system, but the effort would outweigh any advantage

- Some vendors are developing large JT cryocoolers, but none currently available

\subsection{Liquid nitrogen cooling}

Liquid nitrogen cooling has several distinct advantages. The temperature difference between the nitrogen and the focal plate is large enough to allow small flexible thermal links. Liquid nitrogen has been used successfully many times in the past. Existing cameras at CTIO and other locations use manually filled liquid nitrogen reservoirs. At 168 liters volume the vessel size required for daily fills was too large to fit in the space allocated. The cost of nitrogen at CTIO is high. 
Nitrogen circulation has the advantages of stable temperature, low vibration and low space usage near the camera. It has the disadvantage of more equipment including vacuum insulated hoses, a pump and a vessel.

\section{Nitrogen Circulation}

\subsection{Subcooled, saturated or Superheated}

Subcooled liquid, saturated and superheated gaseous nitrogen circulation were compared. Gaseous nitrogen has the advantages of small inventory so lower Oxygen Deficiency Hazard (ODH) and no risk of vibrations due to two phase flow. The disadvantages are a much larger camera heat exchanger and increased risk of temperature instability. Subcooled liquid could be used if testing shows that two-phase flow separation causes too much vibration. It has the disadvantages of required a more complex piping and control system and might suffer cavitation at the heat exchanger.

\subsection{Saturated liquid circulation details}

Saturated liquid circulation was chosen as the most temperature stable fluid condition. Saturation pressure at the camera heat exchanger can be easily measured and controlled regardless of telescope elevation. Two-phase flow pressure drop calculations and flow regime mapping were used to select pipe sizes. To decrease bubble size at the highest point, the camera heat exchanger, the operating pressure was increased to $0.76 \mathrm{MPa}$. This pressure is easily contained with ordinary piping components. To reduce the fluid quality at the camera heat exchanger the pump vessel will be installed at the highest convenient location in the dome. All of the refrigerator contingency was assumed to be located at the camera vessel, which is a worst case scenario.

Nitrogen system design basis

- Operating pressure $0.76 \mathrm{MPa}$

- Operating temperature $100 \mathrm{~K}$

- Flow rate 226 grams/second (7.5 1/min)

- 200 watts camera heat load, including contingency

- Pump elevation 12 meters below camera top

Calculations of thermodynamic conditions were made at notable points along cooling circuit, taking into account heat loads, piping pressure drop and elevation. The points are depicted in Figure 2 and the results listed in table 4. Piping pressure drop and Bernoulli's equation were used to find pressure and quality at points along the circulation path. Frictional Pressure drop was found using Darcy's equation ${ }^{2}$ with two-phase corrections from "Boiling Heat Transfer and Two-Phase Flow". 
Figure 2, Process Flow Sheet

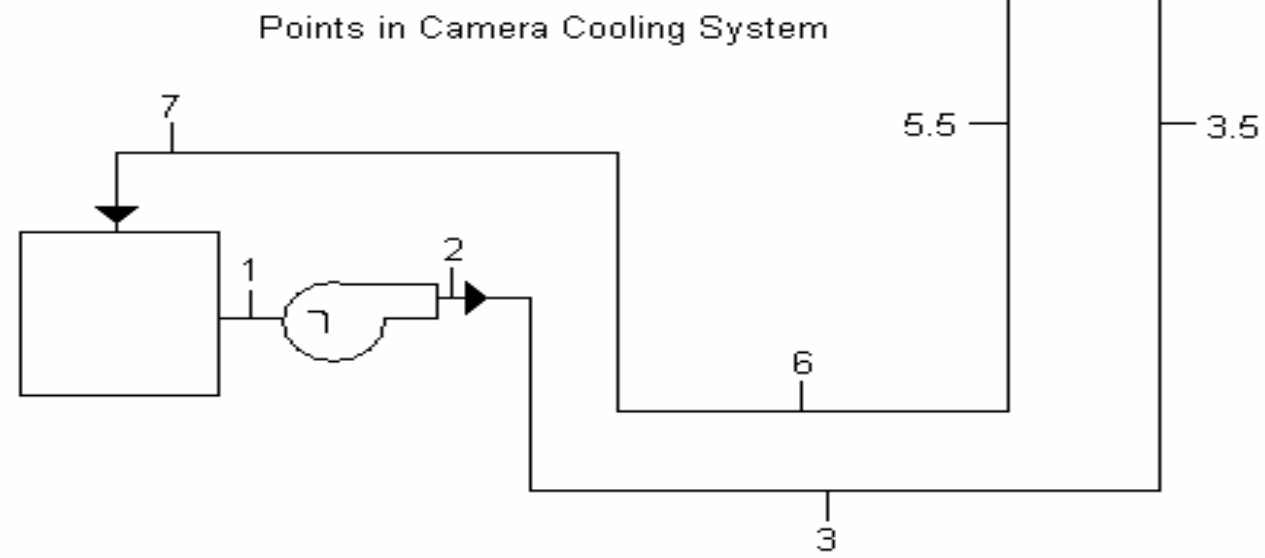

Table 4, Thermodynamic conditions at specific points

\begin{tabular}{|c|c|c|c|c|c|c|c|c|}
\hline Conditions included & Point & $\begin{array}{c}\text { Temp } \\
(\mathrm{K})\end{array}$ & $\begin{array}{c}\text { Pressure } \\
(\mathrm{MPa})\end{array}$ & $\begin{array}{c}\text { Bulk } \\
\text { Density } \\
\left(\mathrm{g} / \mathrm{m}^{\wedge} 3\right)\end{array}$ & $\begin{array}{l}\text { Liquid } \\
\text { Density } \\
\left(\mathrm{g} / \mathrm{m}^{\wedge} 3\right)\end{array}$ & $\begin{array}{c}\text { Vapor } \\
\text { Density } \\
\left(\mathrm{g} / \mathrm{m}^{\wedge} 3\right)\end{array}$ & $\begin{array}{c}\text { Bulk } \\
\text { Enthalpy } \\
(\mathrm{J} / \mathrm{g})\end{array}$ & $\begin{array}{c}\text { Quality } \\
\text { (g/g) }\end{array}$ \\
\hline & 1 & 99.7 & 0.760 & 691410 & 691410 & Subcooled & -74.002 & Subcooled \\
\hline With pump boost \& energy & 2 & 99.7 & 0.840 & 691580 & 691580 & Subcooled & -73.869 & Subcooled \\
\hline Head pressure increase & 3 & 99.7 & 0.888 & 691900 & 691900 & Subcooled & -73.869 & Subcooled \\
\hline Heat leak and frictional loss & 3 & 99.7 & 0.879 & 691720 & 691720 & Subcooled & -73.825 & Subcooled \\
\hline Head Pressure loss & 3.5 & 99.7 & 0.815 & 691290 & 691290 & Subcooled & -73.825 & Subcooled \\
\hline Heat leak and frictional loss & 3.5 & 99.7 & 0.810 & 691200 & 691200 & Subcooled & -73.803 & Subcooled \\
\hline Head Pressure loss & 4 & 99.4 & 0.745 & 621970 & 693120 & 30586 & -73.803 & 0.00528 \\
\hline Heat leak and frictional loss & 4 & 99.3 & 0.739 & 602760 & 693800 & 30339 & -73.803 & 0.00691 \\
\hline Camera Heat Exchanger & 5 & 99.3 & 0.739 & 545010 & 693800 & 30339 & -72.896 & 0.01248 \\
\hline Head pressure increase & 5.5 & 100.1 & 0.792 & 688.58 & 688.58 & Subcooled & -72.896 & Subcooled \\
\hline Frictional loss & 5.5 & 100.1 & 0.782 & 675.79 & 688.94 & 32.116 & -72.896 & 0.000951 \\
\hline Head Pressure increase & 6 & 100.1 & 0.845 & 688.94 & 688.94 & Subcooled & -72.896 & Subcooled \\
\hline Frictional loss & 6 & 100.1 & 0.839 & 688.9 & 688.9 & Subcooled & -72.896 & Subcooled \\
\hline Head pressure loss & 7 & 100.1 & 0.792 & 688.58 & 688.58 & Subcooled & -72.896 & Subcooled \\
\hline Frictional loss & 7 & 100.1 & 0.787 & 688.55 & 688.55 & Subcooled & -72.896 & Subcooled \\
\hline
\end{tabular}




\subsection{Flow Regime}

Flow regime describes how well the phases are mixed in a pipe. The flow regime is a function of the liquid and vapor density, viscosity, quality and velocity. For a particular fluid higher quality moves the conditions to the left and down, while higher velocity moves conditions upward. For more detail see 'Boiling Heat Transfer and Two-Phase Flow'. ${ }^{3}$ For DECam stratified flow should be avoided since it could lead to vertical slug flow or static gas bubbles at high points. This situation will not occur with the conditions chosen. The worst location in the circulation loop is at point 5, the camera heat exchanger outlet. That point is denoted with the dot below the line (D) in figure 3. All other points are in the dispersed bubble regime or subcooled flow.

Figure 3, Horizontal Flow regime

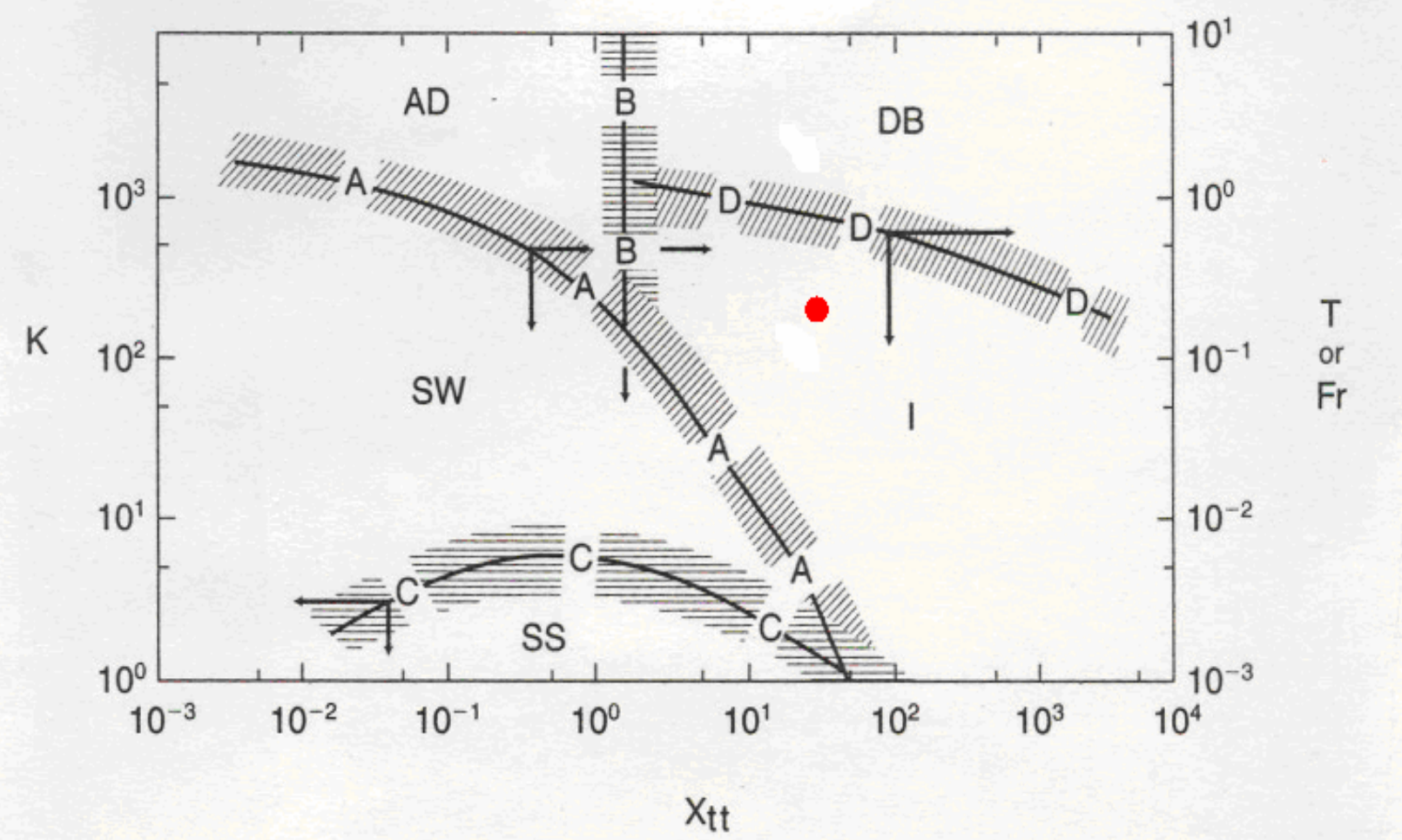

Figure 3.3 Horizontal flow regime map: curves A, B, (Fr) versus $X_{t r}$; curve C, $K$ versus $X_{t r}$; curve D, $T$ versus $X$... (AD. annular dispersed: DB. dispersed bubble: SW. stratified wavv: I. intermittent: SS.

The nitrogen flow direction changes as it passed through the heat exchanger. During operation it may be horizontal, vertical or any orientation. Orientation affects two-phase flow regimes. If the conditions arise where liquid and gas separate and slug flow is present, an unbalance force will occur as slugs of liquid move through the circuit. If this occurs, the unbalance forces will be 0.4 Newton for each 90 degree turn. Measurements of deflection due to gravity as the instrument rotates suggest that this force is negligible.

\subsection{Cryocooler vs. Liquid Deliveries}

The pump dewar can be filled from portable liquid nitrogen supplies and this is a backup in case of cryocooler failure. For the electric power rates and nitrogen supply costs at CTIO, using a cryocooler has much lower utility costs and requires less effort. The cryocooler is expected to operate twelve months without maintenance as opposed to daily nitrogen filling.

\subsection{Cooling Equipment}

The liquid nitrogen pump is a centrifugal pump with a submerged motor. A centrifugal pump was chosen because it is a simple design, easy to operate under a variety of conditions and very reliable. The submerged motor was chosen because it has no shaft seal and it has low static heat leak. The pump is equipped with variable speed drive. It can be run at high speed initially then slowed to half speed for normal operation. Lower speed operation should increase pump bearing 
lifetime. Figure 4 shows the centrifugal pump mounted on its supports. The single stage Gifford-McMahon provides cooling for the circulation system. The compressor and its associated heat rejection will be located outside the dome. The copper nitrogen condenser is attached directly to the cryocooler cold finger and the assembly will be mounted inside the pump dewar. The cryocooler coldhead and condenser are shown in figure 5.

\section{Figure 4 Nitrogen Pump}

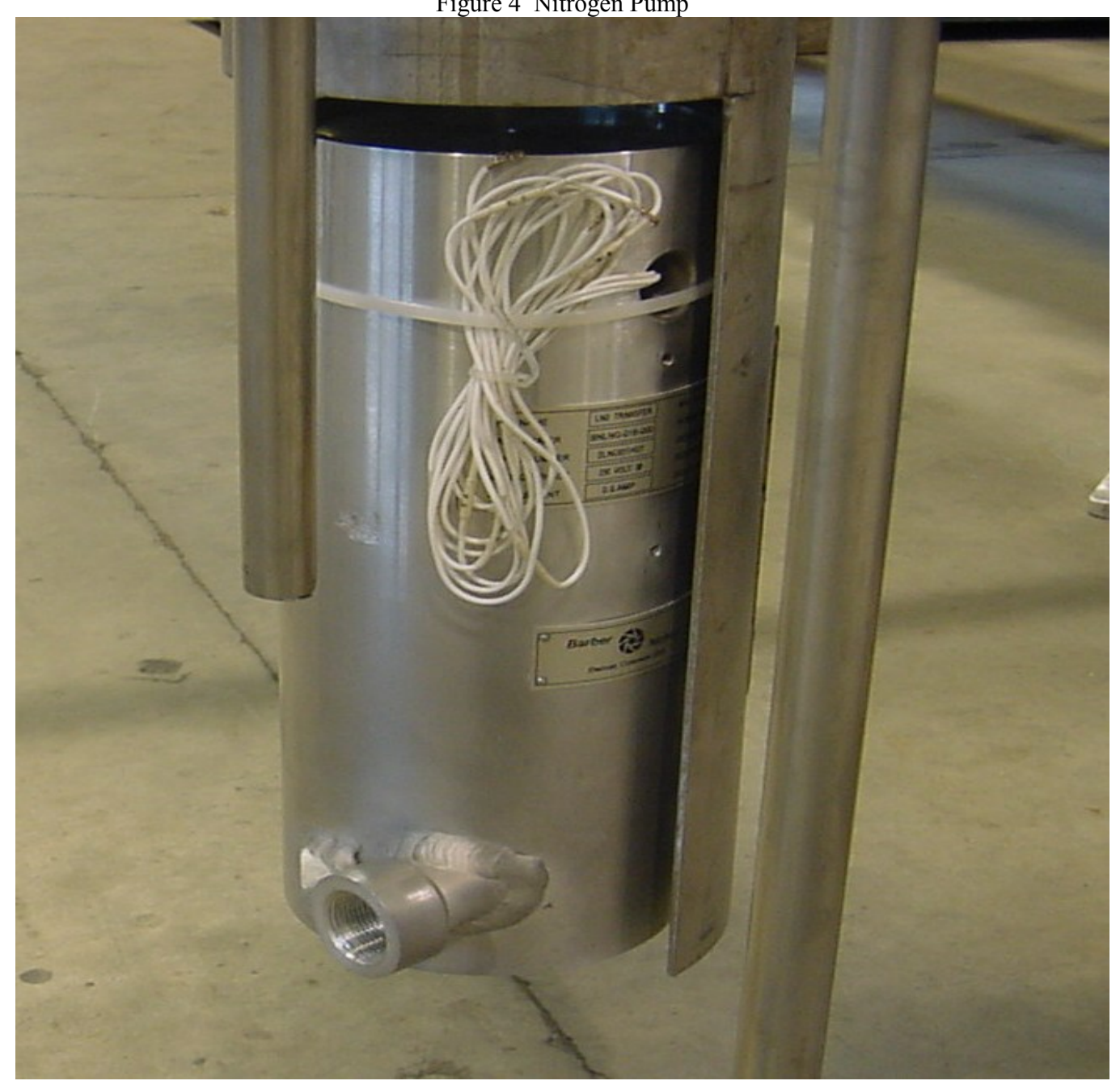


Figure 6 Cryocooler Cold Head and Nitrogen Condenser

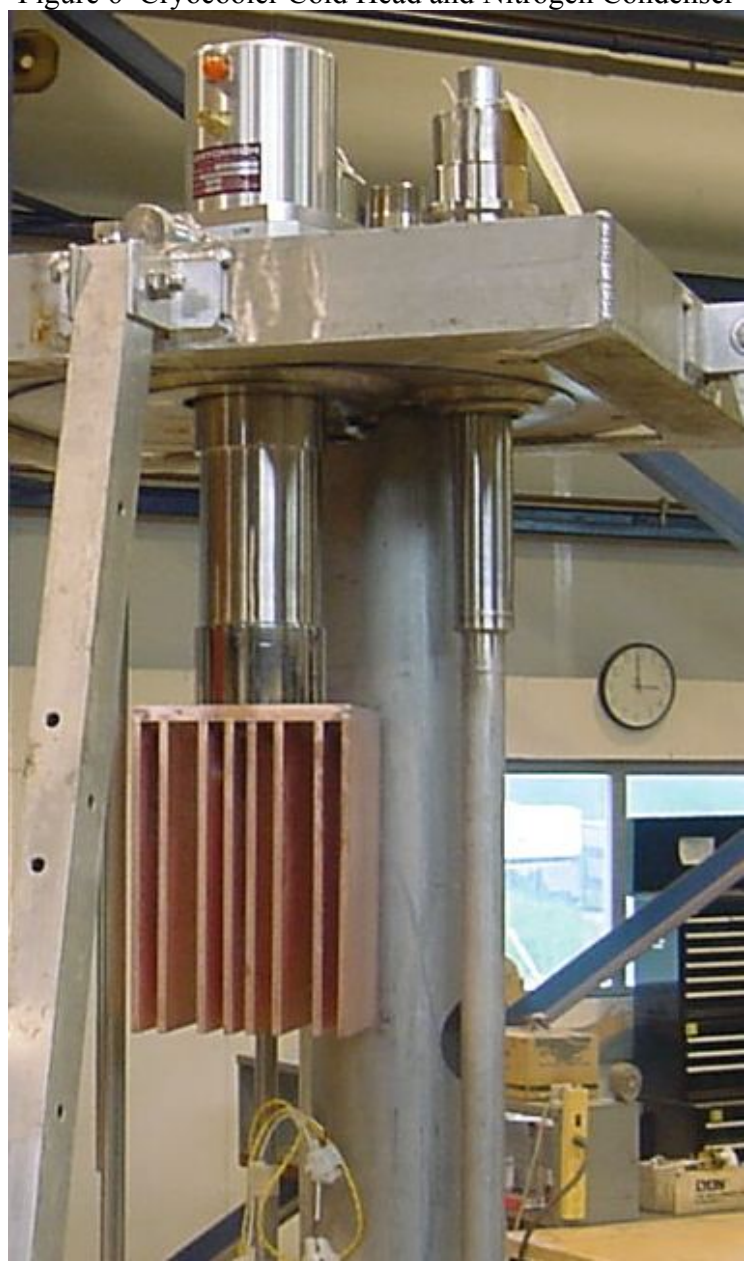

\section{Testing}

A full thermal performance test is planned for August 2008. This test will measure cooling performance and vibration at the camera heat exchanger. The heat exchanger will be located twelve meters above the pump dewar to simulate telescope conditions.

\section{Acknowledgements}

Funding for the DES Projects has been provided by the U.S. Department of Energy, the U.S. National Science Foundation, the Ministry of Science and Education of Spain, the Science and Technology Facilities Council of the United Kingdom, the National Center for Supercomputing Applications at the University of Illinois at UrbanaChampaign, the Kavli Institute of Cosmological Physics at the University of Chicago, Financiadora de Estudos e Projetos, Fundação Carlos Chagas Filho de Amparo à Pesquisa do Estado do Rio de Janeiro, Conselho Nacional de Desenvolvimento Científico e Tecnológico and the Ministério da Ciência e Tecnologia and the Collaborating Institutions in the Dark Energy Survey.

The Collaborating Institutions are Argonne National Laboratories, the University of Cambridge, Centro de Investigaciones Energeticas, Medioambientales y Tecnologicas-Madrid, the University of Chicago, University College 
London, DES-Brazil, Fermilab, the University of Edinburgh, the University of Illinois at Urbana-Champaign, the Institut de Ciencies de l'Espai (IEEC/CSIC), the Institut de Fisica d'Altes Energies, the Lawrence Berkeley National Laboratory, the University of Michigan, the National Optical Astronomy Observatory, the Ohio State University, the University of Pennsylvania, the University of Portsmouth and the University of Sussex.

\section{References}

[1] Flint, E., "Cryocooler Disturbance Reduction with Single and Multiple Axis Active/Passive Vibration Control Systems," Proc SPIE, 3989, 487 (2000)

[2] Crane Engineering Department, [Flow of Fluids Through Valves, Fittings and Pipe], King of Prusssia, PA, Crane Co., $3(1988)$

[3] Tong, L.S., and Tang, Y.S., [Boiling Heat Transfer and Two-Phase Flow], Series in Chemical and Mechanical Engineering, Washington, D.C., Taylor and Francis, 124 (1997) 